\title{
Endovascular Intracranial Micro-Catheter Placement to Treat Cavernous Sinus Thrombosis
}

Shahid M Nimjee*, Honey Jones, Sankalp Gohkale, Carmen Graffagnino, Ali R Zomorodi and Tony P Smith

Department Neurological surgery, The Ohio State University Wexner Medical Center, USA

${ }^{*}$ Corresponding author: Shahid M Nimjee, Department Neurological surgery, The Ohio State University Wexner Medical Center, N-1014 Doan Hall 410 W. 10 th Avenue

Columbus, OH 43235, USA; E-mail: shahid.nimjee@osumc.edu

Received date: July 24, 2015; Accepted date: August 25, 2015; Published date: August 29, 2015

Copyright: $\odot 2015$ Nimjee SM et al. This is an open-access article distributed under the terms of the Creative Commons Attribution License, which permits unrestricted use, distribution, and reproduction in any medium, provided the original author and source are credited.

\begin{abstract}
Cavernous sinus thrombosis (CST) is a rare disease that can have devastating morbidity. Despite medical therapy, in rare circumstances, patients will continue to decline. We present a patient who complained of headache, left eye pain and vision loss. Magnetic resonance imaging revealed bilateral superior ophthalmic vein and cavernous sinus thrombosis. She was treated with antibiotics and intravenous heparin. Despite a therapeutic activated partial thromboplastic time, she progressed to bilateral blindness. Microcatheter transvenous pharmacological thrombolysis with recombinant tissue plasminogen activator (rTPA) was performed including overnight continuous infusion. She recovered light perception the following morning and left the hospital neurologically intact. This case demonstrates that in patients with cerebral deep venous thrombosis that decline despite medical therapy, endovascular intervention is a viable option.
\end{abstract}

Keywords: Cavernous sinus; Superior ophthalmic vein; Thrombus; Endovascular; rTPA

\section{Background}

Cavernous sinus thrombosis (CST) is a rare disease that can have devastating morbidity. The standard treatment for CST is antibiotics and possibly systemic anticoagulation. Despite medical therapy, patients can continue to decline. We present a patient who presented with CST. She was treated with antibiotics and intravenous heparin. Despite a therapeutic activated partial thromboplastic time, she progressed to bilateral blindness, confusion and lethargy. We describe endovascular intervention and thrombolysis, resulting in rapid return of eyesight and baseline neurological function. We also provide a brief overview CST including causes, diagnosis and current treatment.

\section{Case Presentation}

A 71-year-old female with a history of chronic sinusitis complained of worsening headache for a week prior to presentation. She was prescribed antibiotics. Two days later she awoke with left eye pain, proptosis and decreased vision. She presented to an outside hospital and was diagnosed with bilateral cavernous sinus thrombosis by magnetic resonance imaging (MRI). Blood cultures revealed grampositive cocci. Medical treatment included Vancomycin, Zosyn and a continuous infusion of heparin. Despite therapeutic levels by activated partial thromboplastin time (aPTT), she continued to deteriorate neurologically and was transferred to our facility.

On physical examination she moaned but answered yes or no to questions. She followed commands in all four extremities. She had left greater than right eye chemosis and proptosis with complete ptosis of left eye and near-complete of the right (Figure 1A). There were no extra-ocular movements and she was blind in her left eye and could only perceive light in the right.

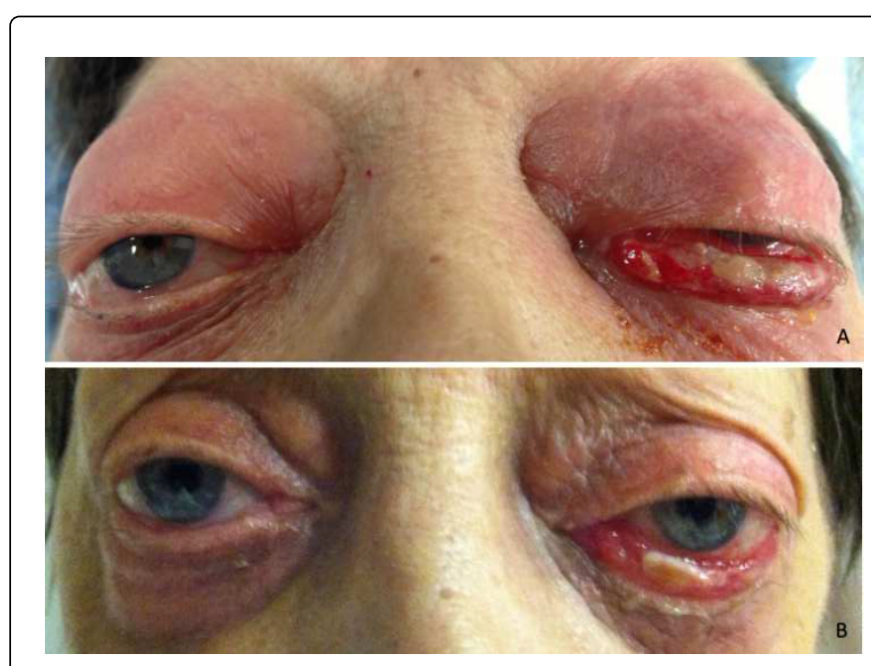

Figure 1: Picture of patient's eyes A. Before intervention after antibiotics and anticoagulation had commenced B. Seven days after intervention.

\section{Treatment}

The right common femoral artery was percutaneously accessed with a micro puncture device and a 5 French introducer sheath was placed. The patient received a bolus of 3000 units of heparin intravenously (IV) and then continued a titrated dose of 2000 units per hour. Her aPTT was 72.7 seconds while on 2000 units per hour.

A cerebral arteriogram was performed, which demonstrated bilateral cavernous sinus thrombosis and left greater than right superior ophthalmic vein thrombosis (Figure 2). The right and left 
femoral veins were then percutaneously accessed with a micro puncture device and a 6 French introducer sheath was placed.
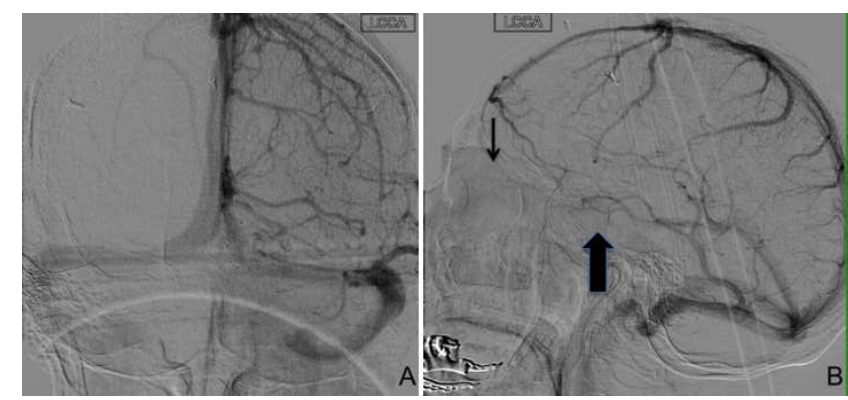

Figure 2: Digital subtraction angiography (DSA) of the venous phase from the left common carotid artery (LCCA) demonstrating patency of venous sinuses and occlusion of superior ophthalmic vein and cavernous sinus. A. Anterior-posterior and B. lateral. The large arrow points towards a void demonstrating no opacification of the cavernous sinus. The small arrow points towards a void demonstrating no opacification of the left superior ophthalmic vein.

A 6 French supporting catheter (Envoy XB, Cordis Inc. Miami Lakes, Florida, USA) was advanced from the right femoral vein into the right internal jugular vein over a 0.035 inch hydrophilic guidewire (Glidewire, Terumo Medical Corp., Somerset New Jersey, USA). A 2 tip micro catheter (Renegade, Boston Scientific Corp. Natick, Massachusetts, USA) was advanced into the right cavernous sinus and right superior ophthalmic vein over a 0.014 inch guidewire (Synchro 2, Stryker Neurovascular, Fremont, California, USA). A right superior ophthalmic vein venogram confirmed thrombosis.

A 6 French Envoy supporting catheter was advanced from the left femoral vein into the left internal jugular and an identical 2 tip micro catheter was advanced over a series of micro wires (Synchro 2 and Synchro Standard, Stryker Neurovascular; Glidewire Gold, Terumo Medical Corp.) into the left cavernous sinus. A left cavernous sinus venogram again confirming thrombosis. Both microcatheters were connected to a continuous infusion system and recombinant thromboplatin activatior (rtPA) (Alteplase, Genetech USA Inc., South San Francisco, California, USA) was administered through the micro catheter as a bolus dose of $4 \mathrm{mg}(2 \mathrm{mg} /$ microcatheter) followed by a 1 $\mathrm{mg}$ per hour infusion (.5 mg/catheter) (Figure 3$)$. The heparin was stopped while the rTPA was being infused.

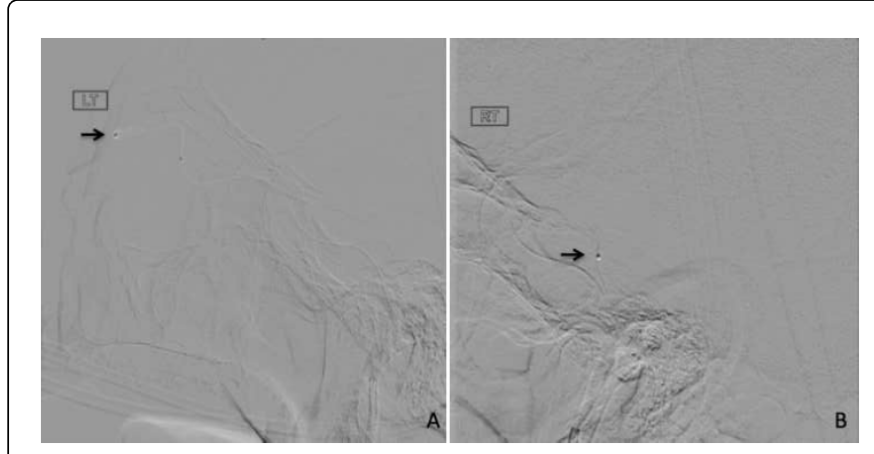

Figure 3: Intraprocedural DSA images of the micro catheter tip position in A. Left superior ophthalmic vein and B. right cavernous sinus.

\section{Outcome and Follow up}

The following morning, the patient's vision improved to the point that she could correctly identify objects in her right eye and had light perception in the left. Computerized tomography (CT) of the brain was performed the following morning demonstrating no evidence of intracranial hemorrhage. All catheters were removed and the IV heparin restarted.

Otolaryngology, head and neck surgery was consulted and recommended endonasal exploration. Surgery revealed sinus erosion consistent with multiple bacterial infections. Two biopsy specimens were consistent with infectious etiology with no evidence of malignancy.

Infectious disease was consulted. They recommended continued antibiotics while in the hospital followed by 3 weeks of intravenous Nafcillin and added Moxifloxacin to cover gram-negative bacteria and anaerobes given the intraoperative inflammation seen.

Hematology was consulted. They recommended therapeutic lovenox for 12 months given the potential interaction of the antibiotics with warfarin.

She was discharge after 7 days with a normal neurological exam. Her vision was 20/20 bilaterally with near-complete resolution of her proptosis (Figure 1B). Her extra-ocular movements were intact. Ten months after treatment, her vision continues to be intact with no new headaches or infections.

\section{Discussion}

Cavernous sinus thrombosis (CST) is a rare pathological manifestation generally seen in aseptic patients who have had endonasal surgery or other trauma or in patients with an infectious source including sinusitis, otitis, odontogenic basis, as well as other infections of the face [1]. The pathophysiology behind the development of CST involves inflammatory changes from regional infection of the eyes generating pathological thrombosis, affecting the angular veins. This then invades the superior ophthalmic veins and directly spreads to the cavernous sinus. It presents with headache, vision changes, chemosis and proptosis. Cranial nerve symptoms, especially III and VI are hallmarks of progression. Imaging studies for diagnosis are most often $\mathrm{CT}$ venogram and MR venogram. In cases 
Citation: Nimjee SM, Jones H, Gohkale S, Graffagnino C, Zomorodi AR, et al. (2015) Endovascular Intracranial Micro-Catheter Placement to Treat Cavernous Sinus Thrombosis. J Neurol Neurophysiol 6: 309. doi:10.4172/2155-9562.1000309

Page 3 of 3

where clinical suspicion exists yet conservative imaging is uncertain, digital subtraction angiography can be useful [1].

Therapy for CST includes endonasal exploration and antibiotics in cases where infection is suspected $[2,3]$. Systemic anticoagulation therapy is employed but their use has never been adequately studied given the rarity of CST [2]. This particular case represents an instance where medical therapy was instituted and the patient continued to decline. Overnight infusion of rTPA within the clot allowed durable thrombolysis to occur and conversion to therapeutic anticoagulation prevented re-thrombosis, over the course of 1- year follow up. Others have reported endovascular occlusion to treat increased intraocular pressure associated with CST [4]. Our description of endovascular intervention was based on previous experience with treating dural venous sinus thrombosis (DVST) where a patient declined neurologically despite maximal medical therapy [5]. As in this case, the patient's neurological exam improved rapidly following endovascular thrombolysis. As opposed to the case report where the patient's vision improved with endovascular superior ophthalmic vein over the course of 4 months[4], our patient's visual complaints resolved in 7 days.

The potential major risk of endovascular intervention is hemorrhage. Given the patient's precipitous decline and after obtaining full consent from the family, we felt this was the only treatment option to avoid significant morbidity.

In conclusion, endovascular treatment of CST is a reasonable option in patients who decline neurologically despite medical management.

\section{References}

1. Desa V, Green R (2012) Cavernous sinus thrombosis: current therapy. Journal of oral and maxillofacial surgery: official journal of the American Association of Oral and Maxillofacial Surgeons 70: 2085-2091.

2. Bhatia K, Jones NS (2002) Septic cavernous sinus thrombosis secondary to sinusitis: are anticoagulants indicated? A review of the literature. See comment in PubMed Commons below J Laryngol Otol 116: 667-676.

3. Lizé F, Verillaud B, Vironneau P, Blancal JP, Guichard JP, et al. (2015) Septic cavernous sinus thrombosis secondary to acute bacterial sinusitis: a retrospective study of seven cases. Am J Rhinol Allergy 29: e7-12.

4. Ladner TR, Davis BJ, He L, Mawn LA, Mocco J (2014) Transorbital superior ophthalmic vein sacrifice to preserve vision in ocular hypertension from aseptic cavernous sinus thrombosis. J Neurointerv Surg .

5. Nimjee SM, Powers CJ, Kolls BJ, Smith T, Britz GW, et al. (2011) Endovascular treatment of venous sinus thrombosis: a case report and review of the literature. J Neurointerv Surg 3: 30-33. 